\title{
Cerebral Oxygen Changes in Neonates During Immediate Transition After Birth and Early Life: An Observational Study
}

This article was published in the following Dove Press journal:

Drug Design, Development and Therapy

\author{
Hang Xue \\ Ziyi Wu \\ Jiaxin Yao \\ Anqi Zhao \\ Lanlan Zheng \\ Xiao Yin (iD) \\ Fang Wang \\ Ping Zhao
}

Department of Anesthesiology, Shengjing Hospital, China Medical University, Shenyang I 10004, People's Republic of China
Correspondence: Ping Zhao

Department of Anesthesiology, Shengjing Hospital, China Medical University, 36

Sanhao Street, Shenyang, People's

Republic of China, II 0004

Tel +86- |894025897|

$\mathrm{Fax}+86-24-23269477$

Email zhaoping_sj@163.com
Purpose: The physiologic transition from a fetus to a neonate is composed of a series of complex processes that include changes in cerebral tissue oxygenation saturation $\left(\mathrm{cSO}_{2}\right)$. Monitoring this process is of great importance. This study aimed to define the $\mathrm{cSO}_{2}$ reference interval in neonates without medical support, extending the measurements until 1 hour after birth, and to determine the incidence of abnormally low or high regional cerebral oxygenation during the neonatal transition.

Patients and Methods: A total of 418 neonates delivered by cesarean section were enrolled. Near-infrared spectroscopy was used to monitor cerebral oxygenation.

Results: We found that $\mathrm{CSO}_{2}$ of the non-oxygen-inhaled intrathecal anesthesia in neonates without medical support increased from about $49.0 \%$ in the second minute. Most of them reached $\mathrm{cSO}_{2}$ relative stabilization at $55.7-81.0 \%$ between 7 and 8 minutes after birth. One hour after birth, newborn $\mathrm{cSO}_{2}$ was maintained at 78.0-87.0\%. The low $\mathrm{cSO}_{2}$ rate among babies born under intrathecal anesthesia with and without maternal oxygen inhalation during cesarean sections was approximately $4.5 \%$ and $9.0 \%$, respectively.

Conclusion: We reported the trend in $\mathrm{cSO}_{2}$ from 2 minutes after birth to 1 hour in the neonatal nursing room and determined the incidence of abnormal regional $\mathrm{cSO}_{2}$ during this neonatal transition period. Anesthesiologists should pay special attention to the risk of $\mathrm{cSO}_{2}$ abnormalities in newborns when managing pregnant women with comorbidities.

Keywords: cerebral oxygenation saturation, transitional period after birth, cesarean section, anesthetic monitoring

\section{Introduction}

The physiologic transitions from a fetus to a neonate include a series of complex processes that show changes in cerebral tissue oxygenation when pulmonary circulation is established after clamping the umbilical cord. ${ }^{1}$ Monitoring this process during immediate transition and into early life is crucial as hypoxia can threaten the metabolic functions of various body systems, particularly the immature neonatal central nervous system. Hypoxia can lead to nerve damage if not corrected in time, and cerebral hypoxia can lead to poor clinical outcomes, such as short-term or long-term brain damage, paralysis, disability, and death. ${ }^{2,3}$ Meanwhile, excessive oxygen exposure can also cause bronchopulmonary dysplasia, retinopathy of neonates, and neurologic damage. ${ }^{4,5}$

Cerebral tissue oxygenation saturation $(\mathrm{cSO} 2)$ can reflect the oxygen delivery and consumption by the brain and is more indicative of brain oxygenation than 
arterial oxygen saturation ( $\mathrm{SpO} 2)$ and heart rate (HR). cSO2 monitoring can guide respiratory and supplemental oxygen support and reduce the risk of cerebral hypoxia during immediate transition and resuscitation after birth. ${ }^{6}$ Near-infrared spectroscopy (NIRS) can measure real-time $\mathrm{CSO}_{2}$ changes in the microcirculation of biologic tissues, including arterioles, venules, and capillaries, noninvasively and approximately $1.2 \mathrm{~cm}$ below the skin surface. The principle of this method is based on the absorption characteristics of oxygenated protein and deoxygenated protein chromophores. ${ }^{7}$ Some studies have described $\mathrm{CSO}_{2}$ changes in the first minutes during the neonatal transition. ${ }^{8-12}$ These neonatologists only studied the range of brain oxygen within 15 minutes after birth, and few of them analyzed the factors that can cause abnormal brain oxygen before birth. ${ }^{13}$ In the present study, our team describes $\mathrm{CSO}_{2}$ changes during the immediate transition until 1 hour after birth after cesarean deliveries. Anesthetic factors, such as oxygen inhalation, or a lack of inhalation, and comorbidities can also affect $\mathrm{cSO}_{2}$ changes during neonatal transition.

Our study aimed to chart the $\mathrm{CSO}_{2}$ changes in neonates without medical support while extending the measurements until 1 hour after birth. We sought to determine the incidence of abnormally low or high regional cerebral oxygenation during neonatal transition.

\section{Patients and Methods \\ Ethics}

This study protocol was approved by the Shengjing Hospital Ethics Committee (No.2016PS125J) and registered in the Chinese Clinical Trial Registry (ChiCTR1900024929). The anesthesiologist informed the research plan during the preoperative visit on the day before the operation and answered the patient's anesthesiarelated questions to relieve any maternal tension before the operation. On admission to the operating room, written informed consent was obtained from eligible patients. In addition, the patients were able to voluntarily participate in, or withdraw from, the experiment. The information obtained was considered confidential, and it was not shared with any third party. This trial was conducted in accordance with the Declaration of Helsinki.

\section{Study Design and Patient Recruitment}

This prospective observational cohort study was conducted at Shengjing Hospital, China Medical University, between
August 3 and December 31, 2019. The enrollment criteria were consecutive full-term neonates (gestational ages between 37 and 42 weeks) born by scheduled cesarean section in the obstetrics department of Shengjing Hospital from pregnant women willing to participate in the study during daytime working hours. The exclusion criteria included (1) mothers with syphilis, hepatitis B infection, HIV infection, or other infectious diseases, (2) fetuses diagnosed with a neurologic brain disease, or those with structural malformations of the frontal brain, scalp, or skull on pregnancy examination, or those with abdominal, heart, and vascular hypoplasia, or any other deformity, and (3) fetuses diagnosed with hemoglobin abnormalities and anemia ahead of surgery.

\section{Data Collection}

Baseline data, including the history of the current pregnancy (maternal body mass index, postmenstrual age [PMA], concurrent obstetric disease, and other disease histories) were collected using a standard questionnaire. Parturient women were instructed to lie in the most comfortable position (either left or right lateral position) to prevent hypotension in the supine position. Physiologic indices on admission to the operating room, but before delivery $\left(\mathrm{SpO}_{2}, \mathrm{HR}\right.$, blood pressure [BP], total hemoglobin), were monitored and recorded.

If the parturient women underwent spinal-epidural anesthesia, several anesthetic factors were assessed, which included positioning the needle puncture into the intervertebral space of the lumbar vertebrae using ultrasound (GE Docking Cart, Type NZCART, USA), administering and dosing the drugs into the subarachnoid space, and measuring the anesthetic levels. Other assessed factors included acupuncture to determine the range of skin analgesia (the upper limits were called anesthetic planes), vasoactive drug administration, and determination of whether inhaled oxygen was given after needle puncture and from the time of subarachnoid administration to umbilical cord clamping. If the mothers received general anesthesia (GA), the time from anesthetic administration to umbilical cord clamping was recorded.

Immediately after birth, umbilical cords were clamped, maternal physiologic indices $\left(\mathrm{SpO}_{2}, \mathrm{HR}, \mathrm{BP}\right)$ were documented, and any special neonatal conditions at birth, including nuchal cords, cord knots, and lack of amniotic fluid, were also documented. After the umbilical cords were clamped, neonates were moved to a radiant warmer and received pressure stroking or oral/nasal suction by the 
neonatologists if the neonates had difficulty breathing owing to particulate meconium, blood clots, thick tenacious mucus, or vernix. A cerebral oximeter monitor (CASMED Fore-sight cerebral oximeter monitor, MC2000, Grenoble, France) with a neonatal sensor was used to measure $\mathrm{cSO}_{2}$ levels. The monitor calculated regional tissue oxygenation using the following formula:

$$
\left(\left[\mathrm{HbO}_{2}\right] /\left[\mathrm{HbO}_{2}+\mathrm{Hb}\right]\right) \times 100 \%
$$

The cerebral oximeter monitor uses a luminescent oxygen sensor to transmit light from the monitor to the patient contact point through a fiber that reduces light-induced heat at the patient end. The monitor captures $\mathrm{cSO}_{2}$ every $2 \mathrm{~s}$. The sensor was placed on the left frontoparietal forehead of each neonate and was secured with a wrap placed around the head. $\mathrm{SpO}_{2}, \mathrm{HR}$, and $\mathrm{BP}$ were measured and recorded using a Mindray monitor (Mindray, BeneVisionN15, China) with a neonatal sensor. Since neonatal skin is tender, BP measurements were tested and recorded once while they were transferred to a radiant warmer after the cord was clamped. Umbilical arterial blood was routinely drawn from the umbilical cord by an obstetrician from which the venous blood gas was analyzed and documented. The time taken for the $\mathrm{cSO}_{2}$ to come to a balanced measurement was determined when the $\mathrm{cSO}_{2}$ floated $<5 \%$ up or down for 1 minute. In addition to this data, the American Society of Anesthesiologists (ASA) physical status classification, Apgar score at 1 and 5 minutes (determined by the neonatologists), and neonatal demographic variables (sex, weight) were recorded by a nurse trained to monitor the neonates.

After initial measurements, the neonates were routinely transferred to the nursing room for a 1-hour observation. The time it took for neonates to be transferred to the nursing room, $\mathrm{SpO}_{2}, \mathrm{HR}, \mathrm{cSO}_{2}$, and other special issues in nursing room, such as spontaneous respiration, supplemental oxygen, or hospitalization were also documented.

\section{Quality Criteria}

$\mathrm{SpO} 2$ is a measure of hemoglobin-oxygen saturation in the arteries and should be greater than $\mathrm{cSO}_{2}$. If $\mathrm{SpO} 2$ was $\leq \mathrm{cSO}_{2}$, both values were discarded from the analysis for detection and elimination of any artifacts, as these were not considered physiologic.

\section{Statistical Analyses and Definitions}

According to the sample size estimation method proposed by Jennen-Steinmetz in $2005,{ }^{14}$ a sample size of 173 would produce a 2 -sided $95 \%$ CI with 0.0125 tolerance error and $90 \%$ test power. About $10 \%$ of neonates in our previous study needed assisted ventilation, and taking into account that $10 \%$ of the data may be missing in the experiment or the exit rate in the experiment, we needed about 220 newborns for $\mathrm{cSO}_{2}$ reference estimation.

The following definitions defined a priori and were used in the data collection and analysis.

Intrathecal anesthesia neonates (IA neonates) refer to the newborns whose mothers underwent combined spinalepidural anesthesia or epidural anesthesia.

$G A$ neonates refer to the newborns whose mothers underwent GA.

Oxygen-inhaled neonates refer to newborns whose mothers inhaled oxygen (50\%) after epidural needle punctures or those whose mothers inhaled oxygen (50\%) before GA induction.

Neonatal transitional period covers the time from umbilical cord clamping to the time when the neonates were transferred to a neonatal nursing room.

The source of the $\mathrm{cSO}_{2}$ reference interval was obtained during data collection from the non-oxygen-inhaled IA (NOIA) without medical support (NOIAWM) neonates, such as respiratory support or additional inhaled oxygen needed after birth.

$\mathrm{cSO}_{2}$ relative stabilization time covers the time from clamping the umbilical cord to $\mathrm{cSO}_{2}$ stabilization (when the $\Delta \mathrm{cSO}_{2}$ of 2 consecutive $\mathrm{cSO}_{2}$ measurements was below $5 \%$ for $1 \mathrm{~min}$ )

The duration of the operating room time refers to the time from clamping the umbilical cord to the time when the neonates left the operating room.

Low $\mathrm{cSO}_{2}$ refers to neonatal $\mathrm{cSO}_{2}$ below the $\mathrm{cSO}_{2}$ reference interval during the neonatal transitional period (for more than $30 \mathrm{~s}$ during the OR time, or for more than 3 minutes during the time in the neonatal nursing room).

High $\mathrm{cSO}_{2}$ refers to the $\mathrm{cSO}_{2}$ neonatal measurements above the $\mathrm{cSO}_{2}$ reference interval during the neonatal transitional period (for more than $30 \mathrm{~s}$ in the operating room, or for more than 3 minutes in the neonatal nursing room).

Descriptive summary statistics of the perioperative data for the patient population were presented as frequencies and percentages for the categorical variables and means and SDs for the continuous variables. For the defined variables, including low $\mathrm{cSO}_{2}$, high $\mathrm{cSO}_{2}$, and the $\mathrm{cSO}_{2}$ stabilization time (as defined above), incidence rates were reported as medians (ranges) or 95\% confidence intervals (CI)s. For the 
risk factor analysis of abnormal $\mathrm{cSO}_{2}$ measurements, univariate regression models were run when the incidence was not rare (greater than 5\%). A 2-sample $t$ test was used for continuous variables, and the chi-square test or Fisher exact test was used for categorical variables, as found appropriate. A $P$-value of $<0.05$ was considered statistically significant.

\section{Results}

\section{Patient Population}

Between August 3 and December 31, 2019, a total of 437 pregnant women met the inclusion/exclusion criteria and were informed about the project. Four hundred and twentyone women signed the consent and were enrolled in the study. Cerebral oxygenation saturation monitoring was performed in 421 neonates (418 patients were finally included, and 3 patients had incomplete data), 391 IA neonates, and 27 GA neonates. Three neonates (who underwent tracheal intubation owing to respiratory distress, were directly hospitalized, and did not complete the whole study) were excluded because of inadequate data. All mothers of the GA neonates inhaled oxygen (50\%) before general anesthetic induction. A total of 418 neonates were included in the final data analysis (Figure 1). Among the neonates under non-oxygeninhaled intrathecal anesthesia, 16 newborns received oxygen by mask after assessment by a neonatologist (owing to turbid amniotic fluid and other factors). Cerebral oxygenation saturation curve reference intervals were developed from 230 NOIA neonates who did not require medical support.

No significant differences were found in the characteristics of the baseline population between the enrolled pregnant women and those not enrolled (Table 1). The demographic information of the enrolled population is shown in Table 1.

\section{Anesthesia-Related Data in the Operating Room and Nursing Room}

Of the 418 pregnant women and neonates who completed the study, 391 received IA, and 385 received combined spinal-epidural anesthesia, and 6 received epidural anesthesia. Twenty-seven pregnant women received GA, of which 1 had changed to GA after inadequate analgesia

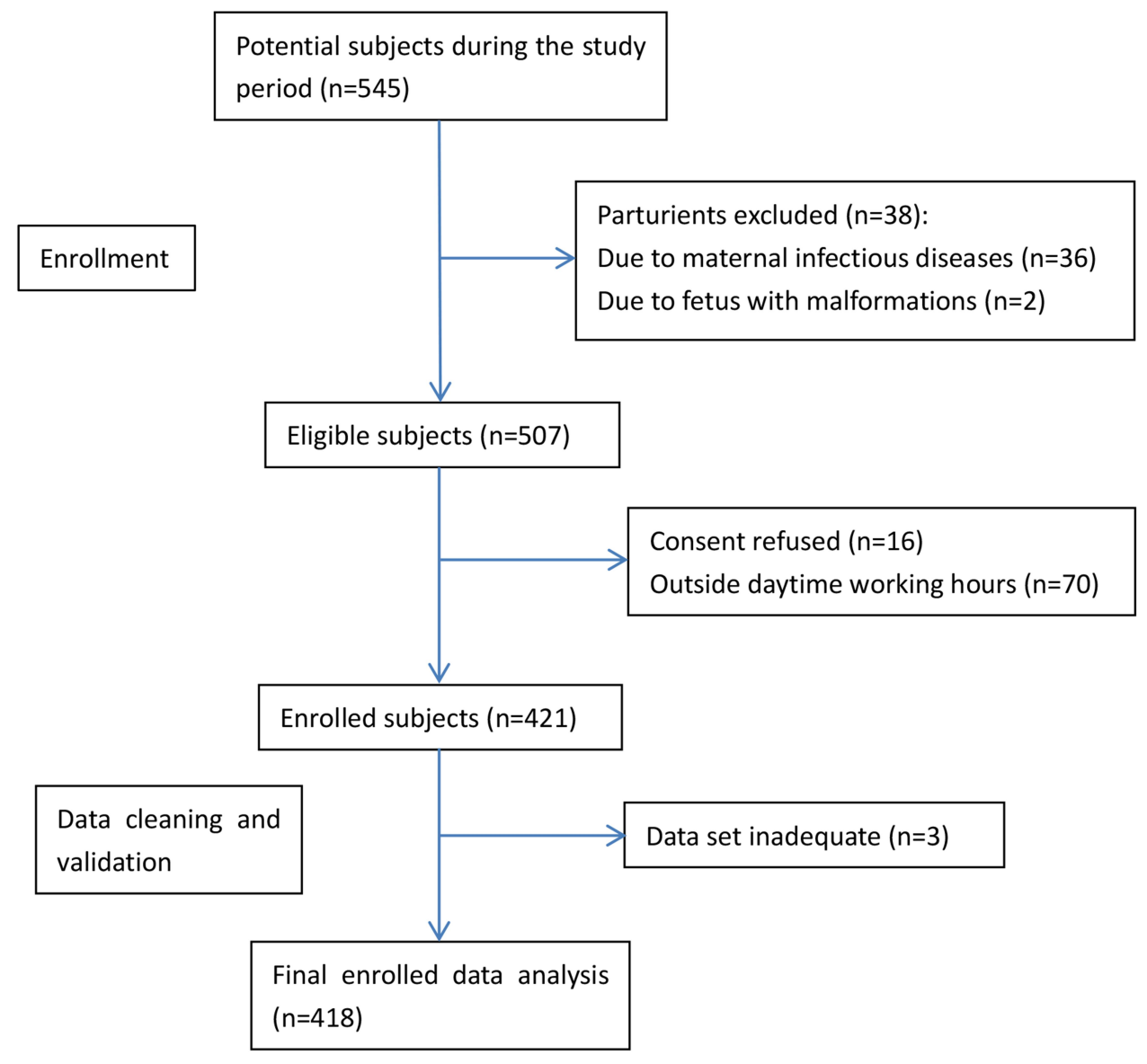

Figure I Flow chart of the study. 
Table I Demographic Information of Enrolled and Not Enrolled Population

\begin{tabular}{|c|c|c|c|c|c|}
\hline $\begin{array}{l}\text { Parturients/Neonates } \\
\text { Characteristics }\end{array}$ & $\begin{array}{l}\text { Not Enrolled } \\
(n=91)\end{array}$ & $\begin{array}{l}\text { Final Enrolled } \\
(n=4 \mid 8)\end{array}$ & $\begin{array}{l}\text { NOIA } \\
(n=246)\end{array}$ & OIA $(n=145)$ & GA(n=27) \\
\hline Parturients age (mean $\pm S D$, years) & $32.25 \pm 5.12$ & $32.30 \pm 4.5 I$ & $32.27 \pm 4.31$ & $32.65 \pm 4.48$ & $30.67 \pm 6.08$ \\
\hline Parturients height (mean $\pm \mathrm{SD}, \mathrm{cm}$ ) & $162.24 \pm 4.60$ & $162.34 \pm 5.28$ & $163.08 \pm 4.93$ & $161.38 \pm 5.49$ & $160.80 \pm 6.13$ \\
\hline Parturients weight (mean \pm SD,kg) & $76.12 \pm 12.60$ & $76.54 \pm 12.25$ & $76.44 \pm 11.45$ & $76.77 \pm 13.16$ & $76.22 \pm|5.5|$ \\
\hline Parturients BMI (mean $\left.\pm \mathrm{SD}, \mathrm{kg} / \mathrm{m}^{2}\right)$ & $28.85 \pm 4.48$ & $29.01 \pm 4.37$ & $28.73 \pm 3.99$ & $29.41 \pm 4.76$ & $29.47 \pm 5.44$ \\
\hline Postmenstrual age (mean $\pm \mathrm{SD}$, days) & $268.91 \pm 9.49$ & $269.67 \pm 8.05$ & $269.76 \pm 8.10$ & $269.58 \pm 7.81$ & $269.27 \pm 9.25$ \\
\hline \multicolumn{6}{|l|}{ ASA physical status (n, \%) } \\
\hline I & $10(\mathrm{II} .0 \%)$ & $50(\mid 2.0 \%)$ & 28 (II.4\%) & $19(13.1 \%)$ & $3(11.1 \%)$ \\
\hline II & 77 (84.6\%) & $358(85.6 \%)$ & $212(86.2 \%)$ & 124 (85.5\%) & $22(81.48 \%)$ \\
\hline III & $4(4.4 \%)$ & $10(2.4 \%)$ & $6(2.4 \%)$ & $2(1.4 \%)$ & $2(7.41 \%)$ \\
\hline IV & $0(0 \%)$ & $0(0 \%)$ & $0(0 \%)$ & $0(0 \%)$ & $0(0 \%)$ \\
\hline \multicolumn{6}{|l|}{ Neonates gender (n, \%) } \\
\hline Male & $40(44.0 \%)$ & $208(49.8 \%)$ & $126(51.2 \%)$ & 70 (48.3\%) & 12 (44.4\%) \\
\hline Female & $5 \mathrm{I}(56.0 \%)$ & $210(50.2 \%)$ & $120(48.8 \%)$ & $75(51.7 \%)$ & 15 (55.6\%) \\
\hline Neonates weight (mean \pm SD, g) & $3273.52 \pm 544.67$ & $3269.44 \pm 484.37$ & $3261.36 \pm 476.34$ & $3280.94 \pm 496.73$ & $3099 \pm 537.80$ \\
\hline
\end{tabular}

Abbreviations: NOIA, non-oxygen-inhaled intrathecal anesthesia; OIA, oxygen-inhaled intrathecal anesthesia; GA, general anesthesia.

from an epidural. Apgar scores immediately after birth were above 5 in all the enrolled neonates. Among the IA neonates, 11 had a score below 10 . Four had scores below 10 in the GA neonates. Apgar scores immediately after birth were significantly different between the GA and IA neonates $(P<0.01)$. The results of other anesthesia-related and neonatal variables are described in Tables 2-4.

\section{Cerebral Oxygen Saturation in Neonates During the Transitional Period}

The $\mathrm{CSO}_{2}$ chart of NOIAWM neonates was drawn 2 minutes after birth (from $49 \%$ [29.5-64.5], median $\left[10^{\text {th }}-90^{\text {th }}\right.$ percentiles]) (Figure 2, Table 5). Figure 2 shows the $10^{\text {th }}, 50^{\text {th }}$, and $90^{\text {th }}$ percentiles of $\mathrm{cSO}_{2}$ values in the NOIAWM neonates, demonstrating that 99 of these neonates $(43.0 \%)$ reached a brain oxygen saturation relative stabilization by $7.3 \mathrm{~min}-$ utes after birth. One hundred and eighty-one of the NOIAWM neonates (78.7\%) achieved brain oxygen saturation balance 8 minutes after birth, and of these, 94 (40.9\%) showed a curve decline of more than $5 \%$ for greater than 30 $\mathrm{s}$ in the operating room. In addition, $37.4 \%$ of the declines in the neonatal $\mathrm{CSO}_{2}$ curve occurred within 2-5 minutes (Table $6)$. The average duration of $\mathrm{cSO}_{2}$ monitoring was 8.8 minutes in the operating room, 43.7 minutes in the nursing room, and 8.0 minutes for transfer from the operating room to the nursing room. Eighty percent of the neonates maintained brain oxygen levels between $55.7 \%-81.0 \%, 7$ minutes after birth before leaving the operating room, and $80 \%$ of the neonates maintained brain oxygen levels between $78.0 \%$ $87.0 \%, 60$ minutes after birth in the neonatal nursing room.

\section{The Occurrence of Low/High $\mathrm{cSO}_{2}$ Levels During the Neonatal Transition Period}

According to the $\mathrm{cSO}_{2}$ values of the NOIAWM neonates, we defined the neonatal $\mathrm{cSO}_{2}$ reference interval at each time point. Table 7 displays the incidence of low or high $\mathrm{cSO}_{2}$ values during the neonatal transition period. Eleven $(4.5 \%)$ of the NOIA neonates had $\mathrm{cSO}_{2}$ values below the reference interval for more than $30 \mathrm{~s}$ in the operating room and more than 3 minutes in the neonatal nursing room. The incidence of low $\mathrm{cSO}_{2}$ levels in OIA neonates and GA neonates were $13(9.0 \%)$ and $6(22.2 \%)$, respectively. Fifteen $(6.1 \%)$ of the NOIA neonates had $\mathrm{cSO}_{2}$ levels above the reference interval for greater than $30 \mathrm{~s}$ in the operating room and more than 3 minutes in the neonatal nursing room. The incidence of high $\mathrm{cSO}_{2}$ levels in OIA neonates and GA neonates were $8(5.5 \%)$ and $5(18.5 \%)$.

\section{Maternal Factors Associated with the Occurrence of Low/High $\mathrm{cSO}_{2}$ Levels}

Factors associated with low and high $\mathrm{cSO}_{2}$ levels during the neonatal transition period were assessed using univariate analyses (Table 8). The factors with odds ratios $>3$ for low $\mathrm{cSO}_{2}$ levels were maternal comorbidities, and ASA III status, while those with odds ratios $>3$ for high $\mathrm{cSO}_{2}$ levels 
Table 2 Perioperative Anesthesia-Related Data of Study Population

\begin{tabular}{|c|c|c|c|c|}
\hline Parturients/Neonates Characteristics & $\begin{array}{l}\text { Final Enrolled } \\
(n=4 \mid 8)\end{array}$ & NOIA $(n=246)$ & OIA $(n=145)$ & GA $(n=27)$ \\
\hline Parturients BP after entering OR (mean $\pm \mathrm{SD}, \mathrm{mmHg}$ ) & $|27.07 \pm| 8.66 \mid$ & $|26.1| \pm \mid 8.09 / 78.34$ & $\mid 27.14 \pm 19.20 /$ & $|36.58 \pm| 8.88 / 87.8 \mid$ \\
\hline & $81.05 \pm 41.83$ & \pm 13.18 & $84.52 \pm 68.59$ & \pm 13.62 \\
\hline $\begin{array}{l}\text { Parturients HR after entering OR (mean } \pm S D \text {, times/ } \\
\text { min) }\end{array}$ & $89.96 \pm 13.87$ & $89.08 \pm 13.90$ & $92.12 \pm 13.05$ & $86.78 \pm 16.86$ \\
\hline Parturients $\mathrm{SpO}_{2}$ after entering $\mathrm{OR}$ (mean $\pm \mathrm{SD}$, \%) & $97.76 \pm 1.28$ & $97.83 \pm 1.32$ & $97.54 \pm 1.20$ & $98.31 \pm 1.29$ \\
\hline Preoperative THb (mean \pm SD, g/L) & $123.18 \pm \mid 1.06$ & $124.30 \pm 10.42$ & $122.3 \pm 10.47$ & $117.62 \pm 17.20$ \\
\hline Comorbidities $^{\mathrm{a}}$ (n, \%) & 154 (36.84\%) & 87 (35.37\%) & 49 (33.79\%) & $18(66.67 \%)$ \\
\hline \multicolumn{5}{|l|}{ Anesthesia level (n, \%) } \\
\hline $\mathrm{T} 4$ & - & $14(5.69 \%)$ & II (7.59\%) & - \\
\hline T5 & - & $3(1.22 \%)$ & $2(1.38 \%)$ & - \\
\hline T6 & - & $143(58.13 \%)$ & $85(58.62 \%)$ & - \\
\hline $\mathrm{T7}$ & - & $9(3.66 \%)$ & 7 (4.83\%) & - \\
\hline T8 & - & $73(29.67 \%)$ & 37 (25.52\%) & - \\
\hline T9 & - & I (0.4I\%) & $0(0 \%)$ & - \\
\hline TIO & - & $3(1.22 \%)$ & $3(2.07 \%)$ & - \\
\hline \multicolumn{5}{|l|}{$\begin{array}{l}\text { Whether given vasoactive drugs before cord } \\
\text { clamping }(\mathrm{n}, \%)\end{array}$} \\
\hline Phenylephrine & 105 (25.12\%) & $66(26.83 \%)$ & $39(26.90 \%)$ & $0(0 \%)$ \\
\hline Ephedrine & 120 (28.7I\%) & $73(29.67 \%)$ & 47 (32.4I\%) & $0(0 \%)$ \\
\hline $\begin{array}{l}\text { Length of time from administration to cord clamping } \\
\text { (mean } \pm \mathrm{SD}, \mathrm{min} \text { ) }\end{array}$ & $12.50 \pm 4.56$ & $12.46 \pm 2.73$ & $13.76 \pm 5.82$ & $5.58 \pm 4.24$ \\
\hline \multirow[t]{2}{*}{ Maternal BP while cord clamping (mean $\pm \mathrm{SD}, \mathrm{mmHg}$ ) } & II $0.96 \pm 22.50 /$ & $110.96 \pm 22.501 /$ & III. $53 \pm 23.40 /$ & $|34.42 \pm 21.5|^{* * \#} /$ \\
\hline & $67.93 \pm 17.33$ & $67.93 \pm 17.33$ & $67.12 \pm 17.90$ & $88.19 \pm 19.25$ \\
\hline $\begin{array}{l}\text { Maternal HR while cord clamping (mean } \pm S D \text {, times/ } \\
\text { min) }\end{array}$ & $82.26 \pm 17.07$ & $82.13 \pm 17.05$ & $81.05 \pm 16.47$ & $89.46 \pm 19.27$ \\
\hline Maternal $\mathrm{SpO}_{2}$ while cord clamping (mean $\pm \mathrm{SD}$, \%) & $98.36 \pm 1.75$ & $97.92 \pm 1.54$ & $99.2 \pm 0.99$ & $97.85 \pm 3.98$ \\
\hline \multicolumn{5}{|l|}{ Special circumstances at birth (n, \%) } \\
\hline Umbilical cord around neck & $83(19.86 \%)$ & $48(19.51 \%)$ & $34(23.45 \%)$ & I (3.70\%) \\
\hline Umbilical cord torsion & $4(0.96 \%)$ & $3(1.22 \%)$ & I (0.69\%) & $0(0 \%)$ \\
\hline Umbilical cord true knot & $3(0.72 \%)$ & $2(0.81 \%)$ & I (0.69\%) & $0(0 \%)$ \\
\hline Single umbilical artery & $4(0.96 \%)$ & $2(0.81 \%)$ & $2(1.38 \%)$ & $0(0 \%)$ \\
\hline Apgar score immediate after birth(median, range) & $10(5-10)$ & $10(8-10)$ & $10(5-10)$ & $10(7-10)^{* * \#}$ \\
\hline Apgar score 5 min after birth(median, range) & $10(9-10)$ & $10(9-10)$ & $10(9-10)$ & $10(9-10)$ \\
\hline Neonatal first BP after cord clamping (mean $\pm \mathrm{SD}$, & $60.28 \pm|3.55 / 3| .74$ & $61.03 \pm|3.55 / 31.7|$ & $59.25 \pm|4.03 / 3| .98$ & $59.04 \pm 10.59 / 30.6$ \\
\hline $\mathrm{mmHg})$ & \pm 11.03 & \pm 10.69 & \pm 11.89 & \pm 9.82 \\
\hline $\begin{array}{l}\text { Neonatal first HR after cord clamping (mean } \pm S D \text {, } \\
\text { times/min) }\end{array}$ & $160.19 \pm 18.28$ & $160.00 \pm 18.52$ & $159.83 \pm 17.67$ & $163.58 \pm 19.90$ \\
\hline Neonatal first $\mathrm{SpO}_{2}$ after cord clamping (mean $\pm \mathrm{SD}, \%$ ) & $64.66 \pm 14.83$ & $64.60 \pm|5.3|$ & $65.63 \pm 13.13$ & $60.04 \pm 18.76$ \\
\hline Whether received medical support (n, \%) & $13(3.11 \%)$ & $6(2.44 \%)$ & $6(4.14 \%)$ & I (4.70\%) \\
\hline Operating room dwell time(mean $\pm \mathrm{SD}$, min) & $8.84 \pm 1.71$ & $8.80 \pm 1.67$ & $8.93 \pm 1.87$ & $8.69 \pm 1.29$ \\
\hline
\end{tabular}

Notes: ${ }^{* * P}<0.01$ compared with NOIA group; \#P < 0.01 compared with OIA group; a: Include hypertensive disorder complicating pregnancy, gestational diabetes mellitus, hypothyrea, hashimoto disease, arrhythmia (frequent junctional premature beats, junctional premature beats with differential junctional escape, ventricular extrasystoleventricular), asthma, congenital heart defect, thrombocytopenic purpura, aplastic anemia.

Abbreviations: NOIA, non-oxygen-inhaled intrathecal anesthesia; OIA, oxygen-inhaled intrathecal anesthesia; GA, general anesthesia.

included maternal comorbidities, and maternal systolic BPs above $143 \mathrm{mmHg}$ or diastolic BP above $90 \mathrm{mmHg}$ after IA. We did not identify anesthetic planes above T6, time lengths from administration to umbilical cord clamping of greater than 17 minutes, existing umbilical cord problems, or risk factors for abnormal $\mathrm{cSO}_{2}$ levels. 
Table 3 Umbilical Arterial Blood Gas Analysis

\begin{tabular}{|c|c|c|c|c|}
\hline Blood Gas Parameters & Final Enrolled $(n=418)$ & NOIA $(n=246)$ & OIA $(n=145)$ & GA $(n=27)$ \\
\hline $\mathrm{pH}($ mean $\pm \mathrm{SD})$ & $7.34 \pm 0.04$ & $7.34 \pm 0.03$ & $7.34 \pm 0.04$ & $7.33 \pm 0.03$ \\
\hline $\mathrm{pCO}_{2}($ mean $\pm \mathrm{SD}, \mathrm{mmHg})$ & $47.38 \pm 6.56$ & $47.03 \pm 6.53$ & $47.90 \pm 6.78$ & $48.18 \pm 5.27$ \\
\hline $\mathrm{pO}_{2}($ mean $\pm \mathrm{SD}, \mathrm{mmHg})$ & $21.92 \pm 6.66$ & $21.47 \pm 6.01$ & $21.93 \pm 7.24$ & $25.54 \pm 7.31$ \\
\hline Hct (mean $\pm \mathrm{SD}, \%)$ & $42.13 \pm 4.06$ & $41.82 \pm 3.98$ & $42.45 \pm 4.22$ & $43.3 \pm 3.67$ \\
\hline $\mathrm{HCO}_{3}^{-}$(mean $\left.\pm \mathrm{SD}, \mathrm{mmol} / \mathrm{L}\right)$ & $24.43 \pm 2.15$ & $24.28 \pm 2.03$ & $24.75 \pm 2.37$ & $24.11 \pm 1.84$ \\
\hline $\mathrm{Na}^{+}($mean $\pm \mathrm{SD}, \mathrm{mmol} / \mathrm{L})$ & $135.75 \pm 2.20$ & $135.61 \pm 2.12$ & $135.90 \pm 2.37$ & $136.38 \pm 1.76$ \\
\hline $\mathrm{K}^{+}($mean $\pm \mathrm{SD}, \mathrm{mmol} / \mathrm{L})$ & $4.18 \pm 0.54$ & $4.19 \pm 0.57$ & $4.19 \pm 0.48$ & $3.97 \pm 0.50$ \\
\hline $\mathrm{Ca}^{+}($mean $\pm \mathrm{SD}, \mathrm{mmol} / \mathrm{L})$ & $1.66 \pm 5.33$ & $1.38 \pm 0.09$ & $\mid .47 \pm 0.13 \mathrm{I}$ & $1.43 \pm 0.31$ \\
\hline $\mathrm{Cl}^{-}($mean $\pm \mathrm{SD}, \mathrm{mmol} / \mathrm{L})$ & $107.30 \pm 1.60$ & $107.35 \pm 1.62$ & $107.18 \pm 1.62$ & $107.57 \pm 1.18$ \\
\hline Glu (mean $\pm \mathrm{SD}, \mathrm{mmol} / \mathrm{L})$ & $3.73 \pm 0.57$ & $3.76 \pm 1.62$ & $3.68 \pm 0.57$ & $3.78 \pm 0.65$ \\
\hline Lac (mean $\pm \mathrm{SD}, \mathrm{mmol} / \mathrm{L})$ & $2.20 \pm 0.52$ & $2.22 \pm 0.51$ & $2.20 \pm 0.56$ & $2.03 \pm 0.30$ \\
\hline $\mathrm{tHb}($ mean $\pm \mathrm{SD}, \mathrm{mmol} / \mathrm{L})$ & $|4.92 \pm| .4 \mid$ & $14.84 \pm 1.42$ & $14.99 \pm 1.42$ & $15.34 \pm 1.15$ \\
\hline $\mathrm{SO}_{2}($ mean $\pm \mathrm{SD}, \%)$ & $61.85 \pm 16.90$ & $51.82 \pm 14.47$ & $61.85 \pm 16.90$ & $59.48 \pm 15.74$ \\
\hline $\mathrm{CH}^{+}($mean $\pm \mathrm{SD}, \mathrm{nmol} / \mathrm{L})$ & $45.72 \pm 3.51$ & $45.48 \pm 3.48$ & $45.91 \pm 3.60$ & $46.72 \pm 2.86$ \\
\hline $\mathrm{BE}($ mean $\pm \mathrm{SD}, \mathrm{mmol} / \mathrm{L})$ & $-1.31 \pm 1.59$ & $-1.35 \pm 1.49$ & $-1.18 \pm 1.80$ & $-1.58 \pm 1.40$ \\
\hline
\end{tabular}

Abbreviations: NOIA, non-oxygen-inhaled intrathecal anesthesia; OIA, oxygen-inhaled intrathecal anesthesia; GA, general anesthesia.

Table 4 Nursing Room Data

\begin{tabular}{|l|l|l|l|l|}
\hline Neonates Characteristics & Final Enrolled (n=4I8) & NOIA (n= 246) & OIA (n=I45) & GA (n=27) \\
\hline Neonates transfer time (mean \pm SD, min) & $7.95 \pm 3.70$ & $7.77 \pm 3.69$ & $8.10 \pm 3.70$ & $7.72 \pm 3.70$ \\
Neonatal SpO after entering Nursing room (mean \pm SD, \%) & $95.65 \pm 3.88$ & $96.02 \pm 2.97$ & $95.24 \pm 4.19$ & $94.52 \pm 7.55$ \\
Whether received mask oxygen (n, \%) & $8(1.91 \%)$ & $6(2.44 \%)$ & $2(1.38 \%)$ & $0(0 \%)$ \\
\hline Special circumstances in nursing room (n, \%) & & & & \\
Hypoglycemia & $34(8.13 \%)$ & $28(11.38 \%)$ & $5(3.45 \%)$ & $\mathrm{I}(3.70 \%)$ \\
Apnea & $2(0.48 \%)$ & $1(0.41 \%)$ & $\mathrm{I}(0.69 \%)$ & $0(0 \%)$ \\
Whether hospitalized during watching (n, \%) & $10(2.39 \%)$ & $9(3.66 \%)$ & $\mathrm{I}(0.69 \%)$ & $0(0 \%)$ \\
Nursing room dwell time (mean \pm SD, min) & $43.68 \pm 6.02$ & $43.88 \pm 5.32$ & $43.23 \pm 7.37$ & $44.38 \pm 3.06$ \\
\hline
\end{tabular}

Abbreviations: NOIA, non-oxygen-inhaled intrathecal anesthesia; OIA, oxygen-inhaled intrathecal anesthesia; GA, general anesthesia.

\section{Discussion}

In the present study, we recorded that the cerebral oxygenation of non-oxygen-inhaled intrathecal anesthesia neonates without medical support increased from about $49 \%$ in the second minute, and most of these neonates can reach cerebral oxygen saturation relative stabilization at 55.7\%$81.0 \%$ between 7 and 8 minutes after birth before leaving the operating room. One hour after birth, newborn brain

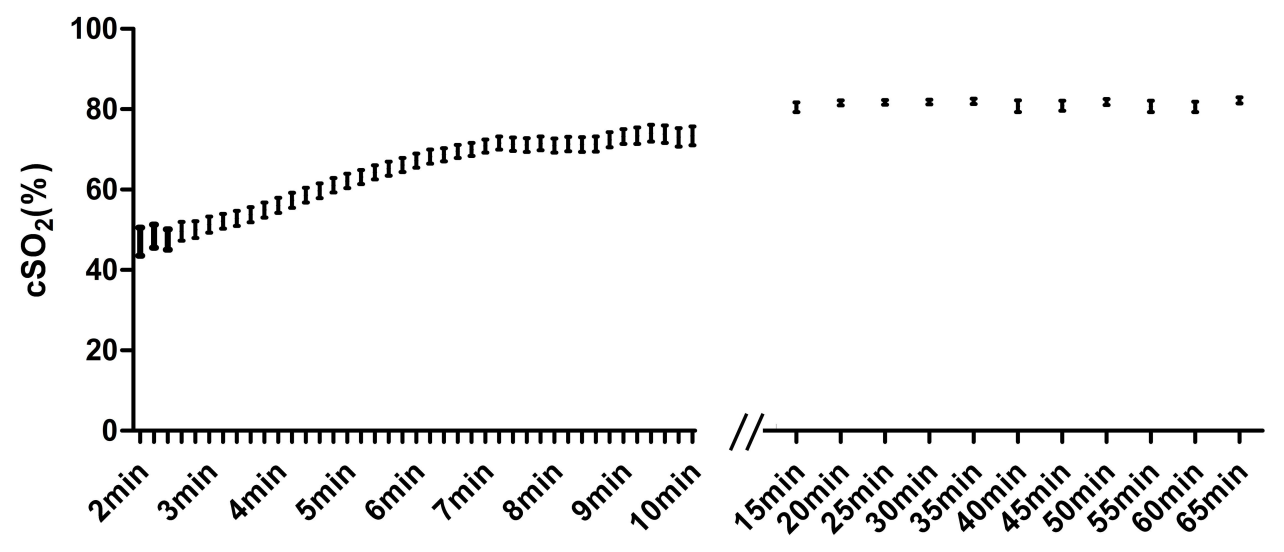

Figure 2 Cerebral regional oxygen saturation reference range of neonates without medical support. 
Table 5 The 10th, 50th, and 90th Percentiles of $\mathrm{CSO}_{2}$ of NOIA Neonates Without Medical Support

\begin{tabular}{|c|c|c|c|}
\hline \multirow[t]{2}{*}{$\min$} & \multicolumn{3}{|c|}{ NOIAWM $(n=230)$} \\
\hline & $10^{\text {th }}$ & $50^{\text {th }}$ & $90^{\text {th }}$ \\
\hline 2 & 29.5 & 49.0 & 64.5 \\
\hline 3 & 34.0 & 51.0 & 69.0 \\
\hline 4 & 38.2 & 57.0 & 74.8 \\
\hline 5 & 44.0 & 64.5 & 77.0 \\
\hline 6 & 49.0 & 70.0 & 80.0 \\
\hline 7 & 54.0 & 73.0 & 82.6 \\
\hline 8 & 55.7 & 73.0 & 81.0 \\
\hline 9 & 61.4 & 75.0 & 82.0 \\
\hline 10 & 62.0 & 74.0 & 83.0 \\
\hline 15 & 73.9 & 80.0 & 86.0 \\
\hline 20 & 76.0 & 82.0 & 86.0 \\
\hline 25 & 76.0 & 82.0 & 87.0 \\
\hline 30 & 77.0 & 82.0 & 87.0 \\
\hline 35 & 77.0 & 82.0 & 86.0 \\
\hline 40 & 74.4 & 81.0 & 86.0 \\
\hline 45 & 73.9 & 81.0 & 87.0 \\
\hline 50 & 78.0 & 82.0 & 86.0 \\
\hline 55 & 75.2 & 81.0 & 86.0 \\
\hline 60 & 73.6 & 81.0 & 87.0 \\
\hline 65 & 78.0 & 83.0 & 87.0 \\
\hline
\end{tabular}

Abbreviation: NOIAWM, non-oxygen-inhaled intrathecal anesthesia neonates without medical support.

oxygen levels were maintained at approximately 78.0$87.0 \%$ in the neonatal nursing room. The low $\mathrm{cSO}_{2}$ rates of babies born under intrathecal anesthesia with or without maternal oxygen inhalation for cesarean sections was approximately $4.5 \%$ and $9.0 \%$, respectively, while the high $\mathrm{cSO}_{2}$ rate was approximately $6.1 \%$ and $5.5 \%$, respectively. Lastly, we identified the maternal factors associated with abnormal cerebral oxygenation during the neonatal transition.

The fetus depends on the placenta for survival; however, neonates can survive independently. The transitional period from intrauterine to extrauterine life includes changes in the circulatory pathways, initiation of ventilation and oxygenation via the lungs instead of the placenta, and many metabolic changes. ${ }^{15}$ The transition period occurs very quickly and is complicated and vulnerable. Most term neonates have reversal flow across the ductus arteriosus (DA) within 10 minutes after birth; meanwhile, systemic vascular resistance also rapidly increases. During this period, these factors and others could cause rapid oxygenation changes in cerebral tissues, and oxygenation in the brain changes faster than that in other tissues. ${ }^{16}$ Neonates with low cerebral oxygenation could suffer from neonatal hypoxic-ischemic brain disease (HIBI) that can cause long-term neurologic sequelae, such as cognitive, behavioral, or memory disorders. ${ }^{17}$ Also, clinical trials show that oxygen overdoses could cause additional oxidative stress-inducing increases in the mortality of neonates during resuscitations. ${ }^{18}$ Excessive oxygen exposure can also cause bronchopulmonary dysplasia, retinopathy of prematurity, and neurologic damage. ${ }^{4,5}$ Therefore, we recorded the $\mathrm{cSO}_{2}$ levels of neonates primarily during the first hour after birth to provide a clear assessment of the normal physiologic indicators during the neonatal transition period to facilitate detection and treatment of abnormal events during this period. Eighty percent of neonates maintained brain oxygen levels of $55.7 \%-81.0 \%, 7$ minutes after birth, and $78.7 \%$ of neonates reached brain oxygen saturation balances between 7 to 8 minutes after birth in the operating room. The reference interval before oxygen saturation relative stabilization and stabilization time points we measured are similar to those reported in studies by Pichler et al ${ }^{11}$ and Baik et al, ${ }^{12}$ and the slight numerical difference may be due to different NIRS devices. Eighty percent neonates maintained brain oxygen levels at $78.0 \%-87.0 \%, 1$ hour after birth in the nursing room. Our study showed significantly extended neonatal $\mathrm{cSO}_{2}$ reference levels and provided effective data references for clinical monitoring.

We reported neonatal $\mathrm{cSO}_{2}$ changes in 230 NOIA neonates without medical support 2 minutes after the umbilical cord was clamped. $\mathrm{cSO}_{2}$ increased rapidly during the first 5 minutes. This general trend was similar to that reported by Pichler et al. ${ }^{11}$ Moreover, we observed an interesting phenomenon, which showed that $60 \%$ of newborns experienced a short period of cerebral oxygen decline between 2 and 5 minutes after cord clamping that lasted approximately 20-50 s. The decreases were not marked, generally between $5 \%$ and $30 \%$, and at the time points where the $\mathrm{cSO}_{2}$ levels fell were relatively scattered, and therefore, the graphic display in Figure 2 is not obvious. Before cord clamping occurs, low-resistance placental tissues are responsible for gas exchange. Also, oxygen-enriched umbilical vein blood flows through the opening foramen ovale and arterial ducts, eventually reaching the left and right ventricles. Then, mixed blood is delivered to the entire body, including the brain. ${ }^{19}$ When the umbilical blood vessel is ligated, systemic circulation resistance sharply increased, and venous blood volumes decreased by $30 \%$ to $50 \%$. $^{20}$ The direction of blood flow is changed by resistance changes in the systemic and 
Table $6 \mathrm{cSO}_{2}$ Related Data During Neonatal Transition Period

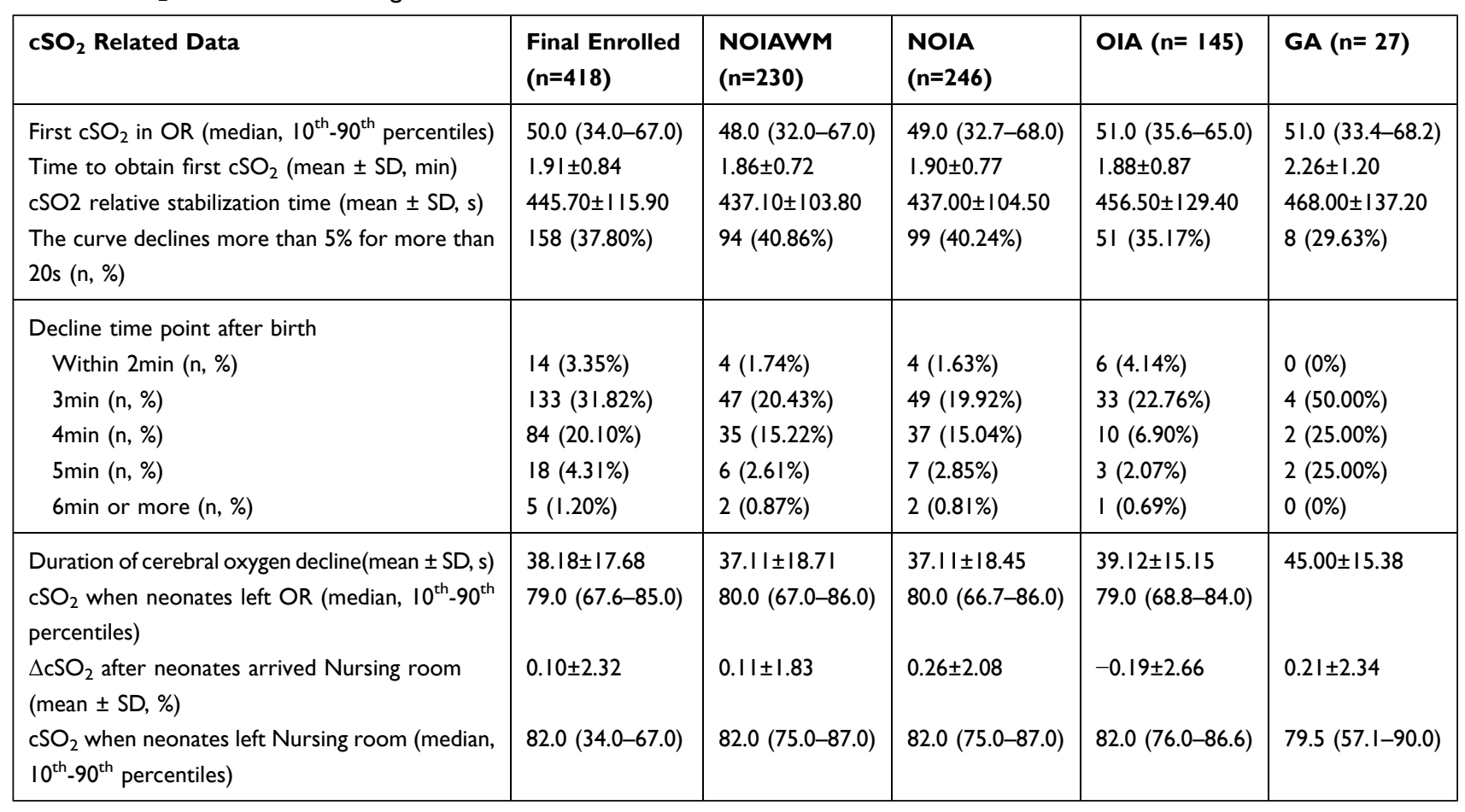

Abbreviations: NOIAWM, non-oxygen-inhaled intrathecal anesthesia neonates without medical support; NOIA, non-oxygen-inhaled intrathecal anesthesia; OIA, oxygeninhaled intrathecal anesthesia; GA, general anesthesia.

Table 7 Incidence of Low/High $\mathrm{CSO}_{2}$ During Neonatal Transition Period Categorized by Anesthesia Type

\begin{tabular}{|c|c|c|c|c|}
\hline Incidence of Low/High $\mathrm{cSO}_{2}$ & Final Enrolled $(\%, 95 \% \mathrm{Cl})$ & NOIA (\%, 95\% Cl) & OIA (\%, $95 \% \mathrm{CI})$ & GA $(\%, 95 \%$ CI) \\
\hline Low $\mathrm{CSO}_{2}$ during neonatal transition period & $\begin{array}{l}7.18(4.70-9.65) \\
n=30\end{array}$ & $\begin{array}{l}4.47(1.89-7.05) \\
n=11\end{array}$ & $\begin{array}{l}8.97(4.3 \mid-13.62) \\
n=13\end{array}$ & $\begin{array}{l}22.22(6.52-37.90)^{* *} \\
n=6\end{array}$ \\
\hline High $\mathrm{cSO}_{2}$ during neonatal transition period & $\begin{array}{l}6.70(4.30-9.10) \\
n=28\end{array}$ & $\begin{array}{l}6.10(2.32-9.87) \\
n=15\end{array}$ & $\begin{array}{l}5.52(1.80-9.23) \\
n=8\end{array}$ & $\begin{array}{l}18.52(4.20-32.83)^{* \#} \\
n=5\end{array}$ \\
\hline
\end{tabular}

Notes: $* \mathrm{P}<0.05$, **P $<0.01$ compared with NOIA group; \#P $<0.05$ compared with OIA group.

Abbreviations: NOIA, non-oxygen-inhaled intrathecal anesthesia; OIA, oxygen-inhaled intrathecal anesthesia; GA, general anesthesia.

pulmonary circulation that promote closure of the anatomic structures associated with vascular shunting, such as the DA and foramen ovale. ${ }^{21}$ Therefore, a slight decrease in neonatal $\mathrm{cSO}_{2}$ levels might be related to systemic vascular resistance, which could decline slightly after sharp $\mathrm{cSO}_{2}$ increases, or because of a decrease in the return of blood volumes or patent DA. We are more inclined to believe that the reduced cerebral oxygenation is related to the open status of the arterial ducts. Clinical trials have proven that the direction and degree of ductal blood flow can influence oxygen delivery to the brain postnatally, and about $72 \%$ of neonates were identified with left-to-right shunts via the DA 15 minutes after birth. ${ }^{21}$ We speculate that this decrease might be related to a decrease in left-to-right shunt traffic via the DA.
Urlesberger et al reported that the incidence of left-toright shunts after birth was larger than that of cerebral oxygen decline measured in our study after cord clamping. We believe that the lack of measurement data from the time of cord clamping to when we measure the first $\mathrm{cSO}_{2}$ value could cause the rate of $\mathrm{cSO}_{2}$ decline to be slightly lower than the true value. Moreover, as $\mathrm{PaCO}_{2}$ has a significant regulatory effect on the cerebrovascular system, we also compared $\mathrm{PaCO}_{2}$ in neonates with and without a decrease in cerebral oxygenation and found no significant differences. Further accurate monitoring of DA blood flow is needed to verify our supposition. Besides, studies show that delayed cord clamping would cause a more stable cardiocirculatory transition and improve cerebral oxygenation. ${ }^{22-24}$ Therefore, a short 
Table 8 Anesthetic and Patient Factors Associated with Low/High $\mathrm{cSO}_{2}$

\begin{tabular}{|c|c|c|}
\hline Anesthetic and Patient Factors & $\begin{array}{l}\text { Low } \mathrm{CSO}_{2} \text { During Neonatal Transition } \\
\text { Period, OR(95\% Cl) }\end{array}$ & $\begin{array}{l}\text { High } \mathrm{cSO}_{2} \text { During Neonatal Transition } \\
\text { Period, OR(95\% Cl) }\end{array}$ \\
\hline Oxygen inhalation & $2.1(0.9-4.8)$ & $0.9(0.4-2.2)$ \\
\hline Comorbidities & $3.2(1.5-7.0)^{* *}$ & $3.4(1.5-7.5)^{* *}$ \\
\hline Anesthesia plane above $\mathrm{T} 6$ & I.I (0.3-4.9) & $0.9(0.9-1.0)$ \\
\hline Vasoactive drug used before cord clamping & $0.6(0.3-1.4)$ & $0.8(0.3-1.9)$ \\
\hline $\begin{array}{l}\text { Maternal systolic BP below } 74 \mathrm{mmHg} \text { or diastolic BP } \\
\text { below } 40 \mathrm{mmHg} \text { under IA }\end{array}$ & $1.8(0.4-8.1)$ & - \\
\hline $\begin{array}{l}\text { Maternal systolic BP above } 143 \mathrm{mmHg} \text { or diastolic } \\
\text { BP above } 90 \mathrm{mmHg} \text { under IA }\end{array}$ & - & $4.8(1.8-12.5)^{* *}$ \\
\hline Maternal $\mathrm{SpO}_{2}$ below $96 \%$ & $3.0(0.8-10.6)$ & - \\
\hline Male sex (male/female) & $0.3(0.9-1.1)$ & $2.0(0.7-6.0)$ \\
\hline ASA II vs I & $3.8(0.5-28.9)$ & $1.9(0.4-8.2)$ \\
\hline ASA III vs I & $21.0(1.9-231.0)^{*}$ & $1.0(0.9-1.0)$ \\
\hline ASA III vs II & $5.5(1.3-22.4)^{*}$ & I.I $(0.1-9.0)$ \\
\hline $\begin{array}{l}\text { Low arteriole pressure(SBP less than } 35 \mathrm{mmHg} \text { or } \\
\text { DBP less than } 11 \mathrm{mmHg} \text { ) }\end{array}$ & $\mathrm{I} .8(0.5-6.1)$ & $0.7(0.3-2.1)$ \\
\hline Low HR(less than 123 times/min) & $3.0(0.3-26.1)$ & $1.6(0.2-13.1)$ \\
\hline $\begin{array}{l}\text { Length of time from administration to cord clamping } \\
\text { more than } 17 \mathrm{~min}\end{array}$ & $1.7(0.2-14.1)$ & $1.3(0.2-10.8)$ \\
\hline Existing problems with umbilical cord at birth & $0.8(0.2-3.9)$ & $0.3(0.0-2.2)$ \\
\hline Neonatal THb less than $14.84 \mathrm{~g} / \mathrm{dL}$ & $0.3(0.1-1.3)$ & $0.7(0.2-2.2)$ \\
\hline Neonatal Glu less than $3.78 \mathrm{mmol} / \mathrm{L}$ & $0.7(0.2-2.1)$ & $2.1(0.7-6.2)$ \\
\hline Neonatal $\mathrm{O}_{2} \mathrm{Hb}$ less than $51.67 \%$ & $1.7(0.3-9.8)$ & $1.4(0.2-8.0)$ \\
\hline Maternal THb less than $124.6 \mathrm{~g} / \mathrm{L}$ & $1.2(0.4-3.3)$ & $0.5(0.1-1.5)$ \\
\hline
\end{tabular}

Notes: **Data show statistical significance at $\mathrm{P}<0.01$; *Data show statistical significance at $\mathrm{P}<0.05$.

period of cerebral oxygen decline between 2 and 5 minutes in our study might also be due to the unstable cardiocirculatory transition caused by early cord clamping, and needs further investigation regarding the cord clamping timing. Moreover, in our study $\mathrm{cSO}_{2}$ increased until minute 7 or 8 , which is similar to the findings in studies by Pichler ${ }^{11}$ and Schwaberger, ${ }^{25}$ where $\mathrm{SpO} 2$ increased until minute 10 or even until minute 12 . We thought that might be explained by a compensatory decrease in cerebral perfusion. Schwaberger et al demonstrated a significant decrease in cerebral blood volume assessed by NIRS in full-term infants after cesarean section. ${ }^{24}$

The secondary outcomes of this study were that low $\mathrm{cSO}_{2}$ rates in newborns born under intrathecal anesthesia cesarean sections with and without oxygen inhalation were approximately $4.5 \%$ and $9.0 \%$, respectively, with no significant difference, and the high $\mathrm{cSO}_{2}$ rates were approximately $6.1 \%$ and $5.5 \%$, respectively, with no significant difference. We also analyzed the correlation between oxygen inhalation during intrathecal anesthesia and abnormal cerebral oxygenation in neonates and found weak correlations. The univariate regression used in this study indicated that low cerebral oxygenation levels were associated with several factors, including maternal comorbidities; further, the ASA III stage and high cerebral oxygenation levels were associated with maternal comorbidities, and maternal systolic BPs above $143 \mathrm{mmHg}$ or diastolic BP above $90 \mathrm{mmHg}$ after IA.

For maternal comorbidities, the risk factors for low cerebral oxygenation in neonates were primarily circulatory disease, and metabolic diseases, such as gestational diabetes mellitus. A study by Cnattingius et al found that the risks of severe asphyxia-related neonatal complications increased in the offspring of mothers with diabetes mellitus. ${ }^{26}$ Hyperglycemia in pregnant women might increase fetal hyperglycemia, and therefore, increase the risk of hypoxia. ${ }^{27,28}$ These findings might explain the relationship between the maternal comorbidities of gestational diabetes mellitus and low cerebral oxygenation. However, we did not find a significant correlation in our regression analysis regarding neonatal hyperglycemia and low cerebral oxygenation, and no correlation was found with the other blood gas-related indicators. This finding is in contrast with Mattersberger's result ${ }^{29}$ that blood glucose 
concentration was associated with cerebral oxygenation during the immediate transition after birth in neonates born at term and preterm. This might be because of the different testing time points between the 2 studies. The neonatal blood glucose concentration in our study was derived from the umbilical arterial blood, while Mattersberger's was tested 10-15 minutes after birth suggesting an influence of metabolic status on cerebral oxygenation after birth transition period. Therefore, in the management anesthesia of cesarean sections for pregnant women with comorbidities, such as diabetes mellitus, anesthesiologists should pay attention to the risk of low cerebral oxygen in newborns.

We also found that neonates born under IA during cesarean sections from mothers with comorbidities, such as pregnancy-induced hypertension (PIH), were more likely to have high cerebral oxygen levels. The pathophysiologic changes involved with PIH mainly include systemic arterial spasms, which can lead to insufficient intrauterine fetal blood supplies or chronic hypoxia. ${ }^{30}$ However, in this study, we found that pregnant women with PIH were at a higher risk of giving birth to neonates with abnormally high cerebral oxygen levels, which might be related to a compensatory increase in total hemoglobin $(\mathrm{tHb})$ levels or red blood cell volumes. ${ }^{31}$ We analyzed the relationship between neonatal tHb levels and high $\mathrm{cSO}_{2}$ rate; however, the regression analyses of the correlations were also not significant. At the same time, of the 418 patients enrolled in this study, 37 pregnant women suffered from PIH. Among these PIH pregnant women, 16 newborns were found to have growth restriction. This is consistent with Baik-Schneditz's research that an intrauterine growth restriction group did show significantly higher $\mathrm{cSO}_{2}$ values. ${ }^{32}$ The abnormal high cerebral oxygen levels might cause oxidative stress and cell damage in oxygensensitive organs, such as the developing brain. ${ }^{32}$ Therefore, anesthesiologists should pay attention to the risk of neonatal cerebral oxygen abnormalities when managing anesthesia in a pregnant mother with comorbidities, such as PIH. In contrast, decreases in BP were shown to poorly correlate with decreases in relative $\mathrm{cSO}_{2}$ levels, which were comparable to the findings of 2 previous studies. $^{33,34}$

We also analyzed the relationship between maternal $\mathrm{SpO}_{2}$, neonatal $\mathrm{BP}$, neonatal $\mathrm{HR}$, other factors, and abnormal cerebral oxygen levels. The maternal and neonatalrelated values listed in Table 7 were derived from the estimated ranges of NOIAWM neonates as the mean \pm
1.96 SD. The correlations were weak. There are several limitations to this study. First, we observed the normal range of cerebral oxygenation within 1 hour after birth and calculated the abnormal low/high cerebral oxygenation rate and analyzed the related factors. Data collection on the long-term outcomes of neonates with abnormal cerebral oxygenation has not been completed, and it is not yet concluded whether there is a correlation between abnormal cerebral oxygenation and long-term cognitive dysfunction. Second, we found that abnormal $\mathrm{cSO}_{2}$ rate in neonates born from pregnant women who received GA (standard GA drug used as recommended ${ }^{35}$ ) was higher, and the Apgar scores immediately after birth in the GA group were lower. There were relatively few GA patients; therefore, the analysis of risk factors related to GA is limited. Further expansion of the sample size of participants under GA is warranted to analyze the reason for this phenomenon. Besides, this result is in contrast with that in the study by Willfurth et al, ${ }^{13}$ though this might be due to the difference in the samples. Only term infants after elective section were included in this study. Vaginal birth and preterm birth could also be included in further studies. Third, immediate cord clamping was performed in all the infants, and this might have led to unstable cardiocirculatory transition. Delayed cord clamping should be considered for further investigation. Fourth, owing to the standard process of transferring neonates from the operating room to the nursing room after birth, cerebral oxygen monitoring had to be interrupted. Subsequent observational clinical studies should extend the observation times within the operating room.

In summary, we reported the trend in cerebral oxygenation from 2 minutes after birth to relatively stable from 7-8 minutes before leaving the operating room, and 1 hour after birth in the neonatal nursing room. We also determined the incidence of abnormal regional cerebral oxygenation during this neonatal transition period. Anesthesiologists should pay special attention to the risk of $\mathrm{cSO}_{2}$ abnormalities in newborns when managing pregnant women with comorbidities.

\section{Abbreviations}

ASA, American Society of Anesthesiologists; SpO2, arterial oxygen saturation; BP, blood pressure; cSO2, cerebral oxygenation saturation; CI, confidence interval; Eph, Ephedrine; GA, general anesthesia; HR, heart rate; IUGR, intrauterine growth restriction; IA, intrathecal anesthesia; NIRS, Near-infrared spectroscopy; NOI 
neonates, non-oxygen-inhaled intrathecal anesthesia neonates; NOIAWM, non-oxygen-inhaled intrathecal anesthesia neonates without medical support; OR, operating room; OIA neonates, Oxygen-inhaled intrathecal anesthesia neonates; PMA, postmenstrual age; Phe, Phenylephrine; PIH, pregnancy-induced hypertension; SO2, saturation of oxygen; $\mathrm{THb}$, total hemoglobin.

\section{Ethical Requirements}

The information obtained was considered confidential, and it was not shared with any third party.

\section{Funding}

This research is supported by grant from the National Natural Science Foundation of China (Nos. 81671311, Nos.81870838), the Key Research and Development Program of Liaoning Province (No.2018225004), Liaoning Province Distinguished Professor Support Program (No.XLYC1802096) and Outstanding Scientific Fund of Shengjing Hospital (No. 201708), and Colleges and Universities Basic Research Project Fund of Liaoning Province (No. LQNK201709).

\section{Disclosure}

The authors declare that they have no conflict of interest.

\section{References}

1. Pichler G, Schmölzer GM, Urlesberger B. Cerebral tissue oxygenation during immediate neonatal transition and resuscitation. Front Pediatr. 2017;5:29. doi:10.3389/fped.2017.00029

2. Kurinczuk JJ, White-Koning M, Badawi N. Epidemiology of neonatal encephalopathy and hypoxic-ischaemic encephalopathy. Early Hum Dev. 2010;86(6):329-338.

3. Zhao P, Ji G, Xue $\mathrm{H}$, et al. Isoflurane postconditioning improved long-term neurological outcome possibly via inhibiting the mitochondrial permeability transition pore in neonatal rats after brain hypoxia-ischemia. Neuroscience. 2014;280:193-203. doi:10.1016/j. neuroscience.2014.09.006

4. Yee M, Buczynski BW, O'Reilly MA. Neonatal hyperoxia stimulates the expansion of alveolar epithelial type II cells. Am J Respir Cell Mol Biol. 2014;50(4):757-766. doi:10.1165/rcmb.2013-0207OC

5. Volpe JJ. Brain injury in premature infants: a complex amalgam of destructive and developmental disturbances. Lancet Neurol. 2009;8 (1):110-124.

6. Pichler G, Urlesberger B, Baik N, et al. Cerebral oxygen saturation to guide oxygen delivery in preterm neonates for the immediate transition after birth: a 2-center randomized controlled pilot feasibility trial. J Pediatr. 2016;170 (1):73-8.e1-73-8.e4. doi:10.1016/j.jpeds.2015.11.053

7. Pellicer A, Bravo Mdel C. Near-infrared spectroscopy: a methodology-focused review. Semin Fetal Neonatal Med. 2011;16 (1):42-49. doi:10.1016/j.siny.2010.05.003

8. Fauchere JC, Schulz G, Haensse D, et al. Nearinfrared spectroscopy measurements of cerebral oxygenation in newborns during immediate postnatal adaptation. J Pediatr. 2010;156:372-376. doi:10.1016/j. jpeds.2009.09.050
9. Fuchs H, Lindner W, Buschko A, Almazam M, Hummler HD, Schmid MB. Brain oxygenation monitoring during neonatal resuscitation of very low birth weight infants. J Perinatol. 2012;32:356-362. doi:10.1038/jp.2011.110

10. Isobe K, Kusaka T, Fujikawa Y, et al. Measurement of cerebral oxygenation in neonates after vaginal delivery and cesarean section using full-spectrum near infrared spectroscopy. Comp Biochem Physiol Part a Mol Integr Physiol. 2002;132:133-138. doi:10.1016/ S1095-6433(01)00539-6

11. Pichler G, Binder C, Avian A, Beckenbach E, Schmölzer GM, Urlesberger B. Reference ranges for regional cerebral tissue oxygen saturation and fractional oxygen extraction in neonates during immediate transition after birth. J Pediatr. 2013;163(6):1558-1563. doi:10.1016/j.jpeds.2013.07.007

12. Baik N, Urlesberger B, Schwaberger B, et al. Reference ranges for cerebral tissue oxygen saturation index in term neonates during immediate neonatal transition after birth. Neonatology. 2015;108 (4):283-286. doi:10.1159/000438450

13. Willfurth I, Baik-Schneditz N, Schwaberger B, et al. Cerebral oxygenation in neonates immediately after cesarean section and mode of maternal anesthesia. Neonatology. 2019;116(2):132-139. doi:10.1159/000499046

14. Jennen-Steinmetz C, Wellek S. A new approach to sample size calculation for reference interval studies. Stat Med. 2005;24 (20):3199-3212. doi:10.1002/sim.2177

15. Morton SU, Brodsky D. Fetal physiology and the transition to extrauterine life. Clin Perinatol. 2016;43(3):395-407.

16. Urlesberger B, Grossauer K, Pocivalnik M, Avian A, Müller W, Pichler G. Regional oxygen saturation of the brain and peripheral tissue during birth transition of term infants. J Pediatr. 2010;157 (5):740-744. doi:10.1016/j.jpeds.2010.05.013

17. Yıldız EP, Ekici B, Tatı B. Neonatal hypoxic ischemic encephalopathy: an update on disease pathogenesis and treatment. Expert Rev Neurother. 2017;17(5):449-459. doi:10.1080/14737175.2017.1259567

18. Torres-Cuevas I, Parra-Llorca A, Sánchez-Illana A, et al. Oxygen and oxidative stress in the perinatal period. Redox Biol. 2017;12:674-681. doi:10.1016/j.redox.2017.03.011

19. Morton SU, Brodsky D. Fetal physiology and the transition to extrauterine life. Clin Perinatol. 2016;43(3):395-407.

20. Crossley KJ, Allison BJ, Polglase GR, Morley CJ, Davis PG, Hooper SB. Dynamic changes in the direction of blood flow through the ductus arteriosus at birth. $J$ Physiol. 2009;587(Pt 19):4695-4704. doi:10.1113/jphysiol.2009.174870

21. Urlesberger B, Brandner A, Pocivalnik M, Koestenberger M, Morris N, Pichler G. A left-to-right shunt via the ductus arteriosus is associated with increased regional cerebral oxygen saturation during neonatal transition. Neonatology. 2013;103(4):259-263. doi:10.1159/000346384

22. Baenziger O, Stolkin F, Keel M, et al. The influence of the timing of cord clamping on postnatal cerebral oxygenation in preterm neonates: a randomized, controlled trial. Pediatrics. 2007;119(3):455-459. doi:10.1542/peds.2006-2725

23. Polglase GR, Blank DA, Barton SK, et al. Physiologically based cord clamping stabilises cardiac output and reduces cerebrovascular injury in asphyxiated near-term lambs. Arch Dis Child Fetal Neonatal Ed. 2018;103;6:530-538.

24. Rabe H, Gyte GM, Díaz-Rossello JL, Duley L. Effect of timing of umbilical cord clamping and other strategies to influence placental transfusion at preterm birth on maternal and infant outcomes. Cochrane Database Syst Rev. 2019;9(9):CD003248.

25. Schwaberger B, Pichler G, Binder-Heschl C, Baik N, Avian A, Urlesberger B. Transitional changes in cerebral blood volume at birth. Neonatology. 2015;108(4):253-258. doi:10.1159/000437347

26. Cnattingius S, Lindam A, Persson M. Risks of asphyxia-related neonatal complications in offspring of mothers with type 1 or type 2 diabetes: the impact of maternal overweight and obesity. Diabetologia. 2017;60(7):1244-1251. doi:10.1007/s00125-0174279-2 
27. Philipps AF, Widness JA, Garcia JF, Raye JR, Schwartz R Erythropoietin elevation in the chronically hyperglycemic fetal lamb. Proc Soc Exp Biol Med. 1982;170(1):42-47. doi:10.3181/ 00379727-170-41394

28. Teramo KA, Widness JA. Increased fetal plasma and amniotic fluid erythropoietin concentrations: markers of intrauterine hypoxia. Neonatology. 2009;95(2):105-116. doi:10.1159/000153094

29. Matterberger C, Baik-Schneditz N, Schwaberger B, et al. Blood glucose and cerebral tissue oxygenation immediately after birth an observational study. J Pediatr. 2018;200:19-23. doi:10.1016/j. jpeds.2018.05.008

30. Thewissen L, Pistorius L, Baerts W, Naulaers G, Van Bel F, Lemmers P. Neonatal haemodynamic effects following foetal exposure to labetalol in hypertensive disorders of pregnancy. $J$ Matern Fetal Neonatal Med. 2017;30(13):1533-1538. doi:10.1080/ 14767058.2016.1193145

31. Hou X, Ding H, Teng Y, Zhou C, Zhang D. NIRS study of cerebral oxygenation and hemodynamics in neonate at birth. Conf Proc IEEE Eng Med Biol Soc. 2011;2011:1229-1232.
32. Baik-Schneditz N, Pichler G, Schwaberger B, et al. Effect of intrauterine growth restriction on cerebral regional oxygen saturation in preterm and term neonates during immediate postnatal transition. Neonatology. 2020;9(6):1-7. doi:10.1159/000507583

33. Michelet D, Arslan O, Hilly J, et al. Intraoperative changes in blood pressure associated with cerebral desaturation in infants. Paediatr Anaesth. 2015;25(7):681-688. doi:10.1111/pan.12671

34. Olbrecht VA, Skowno J, Marchesini V, et al. An, multicenter, observational study of cerebral oxygenation during infant and neonatal anesthesia. Anesthesiology. 2018;128(1):85-96. doi:10.1097/ ALN.0000000000001920

35. Devroe S, Van de Velde M, Rex S. General anesthesia for caesarean section. Curr Opin Anaesthesiol. 2015;28(3):240-246. doi:10.1097/ ACO.0000000000000185

\section{Publish your work in this journal}

Drug Design, Development and Therapy is an international, peerreviewed open-access journal that spans the spectrum of drug design and development through to clinical applications. Clinical outcomes, patient safety, and programs for the development and effective, safe, and sustained use of medicines are a feature of the journal, which has also been accepted for indexing on PubMed Central. The manuscript management system is completely online and includes a very quick and fair peer-review system, which is all easy to use. Visit http://www. dovepress.com/testimonials.php to read real quotes from published authors. 